SLAC-PUB-10432

April 2004

\title{
Soft X-ray precursors of the non-thermal flares in blazars - theoretical predictions
}

\author{
Rafał Moderski ${ }^{1}$, Marek Sikora ${ }^{1,2}$, Greg M. Madejski ${ }^{2}$, and Tuneyoshi Kamae ${ }^{2}$
}

\begin{abstract}
Popular internal shock models, developed to explain production of high energy flares in blazar jets, involve collisions between local overdensities of matter being ejected by a central engine and moving along the jet with different velocities. Prior to such collisions, the matter is relatively cold and therefore does not produce intrinsic non-thermal radiation. However, due to Comptonization of external radiation by cold electrons, the presence of such matter should be apparent by prominent precursor soft X-ray flares, visible prior to non-thermal $\gamma$-ray flares. In this paper we discuss the predicted properties of such precursors and study the dependence of their properties (luminosities and light curves) on kinematic parameters of relativistic ejecta and on an angle of view. We demonstrate that the lack of evidence for luminous soft X-ray precursors can be reconciled with our predictions for their properties if acceleration and collimation of a jet takes about three distance decades. We briefly discuss the severe constraints on the internal shock models that would be imposed by a non-detection of such precursors.
\end{abstract}

Subject headings: galaxies: quasars: general — galaxies: jets — radiation mechanisms: non-thermal — gamma rays: theory — X-rays: general

\section{INTRODUCTION}

Strong jets in radio loud quasars must be launched very deeply in the gravitational potential well, and most likely are powered by rotating black hole and/or innermost parts of an accretion disk. Their formation is very likely mediated by magnetic fields, but the detailed model is not yet established (compare, e.g., scenarios suggested by Contopoulos 1995; Lovelace et al. 1997; Vlahakis \& Königl 2003). In magnetic models, acceleration and

\footnotetext{
${ }^{1}$ Nicolaus Copernicus Astronomical Center, Bartycka 18, 00-716 Warsaw, Poland;

moderski@camk.edu.pl

${ }^{2}$ Stanford Linear Accelerator Center, 2575 Sand Hill Road, Menlo Park, CA 94025, USA
} 
collimation processes are quite smooth and can proceed over several distance decades. It is still not known whether this is accomplished on sub-parsec, parsec, or even larger scales. Vlahakis \& Königl (2003) claim that some VLBI data on the variability of the apparent structure of parsec scale jets are consistent with the acceleration process in the Poynting flux dominated flow. Those authors consider also circular polarization (Homan, Attridge, \& Wardle 2001; Enßlin 2003) and rotation-measure gradients (Asada et al. 2002; Gabuzda \& Murray 2003) to be properties that strongly support dynamical dominance of magnetic fields in parsec scale jets. However, all these features don't have a unique interpretation: accelerating radio features, which are actually observed very rarely, can represent shocks formed on the interface between a jet and a clump of matter entering the jet from outside and being accelerated by the relativistic flow. Likewise, the circular polarization does not necessary require dynamically dominated helical magnetic fields (Ruszkowski \& Begelman 2002). Finally, the gradients in the rotation measure can result from a non-uniform distribution in the external Faraday screen (Homan 2004).

Independent means to verify whether quasar jets are Poynting flux or matter dominated is by studying radiative properties of quasars with jets oriented close to the line of sight. These quasars together with BL Lac objects form the sub-class of active galactic nuclei (AGNs) called blazars. In such objects, any radiation produced within the jet is Doppler boosted into the direction of the observer and dominates over other radiative components of the AGN. As it was pointed out by Begelman \& Sikora (1987) and explored by Sikora \& Madejski (2000), propagation of relativistic jets through very dense radiation fields present in the quasar cores should result in a production of prominent spectral "bumps" in the soft X-ray band due to Comptonization of external UV radiation by cold electrons in a jet. The lack of such features in the observed blazar spectra indicates that the acceleration and collimation of a jet takes place at a distance $\sim 10^{3}$ gravitational radii from the central supermassive black hole. This strongly supports the idea that jets in quasars are, at least initially, magnetically dominated.

The question whether the jets are still magnetically dominated at distances $\sim 0.1-3$ pc, where most of blazar radiation is produced, remains open. The most popular blazar models invoke internal shocks, assumed to be produced by inhomogeneities moving down the jet with different velocities (Sikora et al. 1994; Spada et al. 2001). Since efficient, strong shocks cannot be formed in magnetically dominated jets, in these models the energy flux of relativistic flow is dominated by matter, specifically by protons. Such jets are likely to contain also $\mathrm{e}^{+} \mathrm{e}^{-}$pairs, but with the number $n_{e} / n_{p}<$ tens (as can be inferred from the shock energetics; see Appendix A.1 and A.4), they contribute to the inertia of a jet only negligibly. Prior to their collision, the inhomogeneities are cold, and, as for the case of the cold stationary jet, they upscatter external UV photons up to soft X-ray energies, forming 
X-ray "precursors" which should precede the actual non-thermal flares. Hence, a comparison of the observational data against detailed predictions about the light curves of the soft X-ray precursors and the non-thermal flares can provide an efficient tool to search for an evidence for inhomogeneities, the basic ingredient of the internal shock models (Madejski, Sikora, \& Kamae 2003; Sikora \& Madejski 2002). If such precursors are not detected, it may indicate that non-thermal flares are produced by reconnection events rather than by shocks and, therefore, that jets at parsec-scale distances are still dominated by magnetic fields. At the very least, this can provide the lower limit to the distance where the jets undergo a conversion from the magnetic to matter dominated flows and where shocks could be products of the reconnection events.

In this paper, we develop theoretical framework describing the properties of soft X-ray precursors. We show the dependence of their luminosities on kinematical parameters of ejecta and on the viewing angle, and illustrate how their light curves should compare with the time profiles (including time leads/lags) of the non-thermal flares.

\section{ASSUMPTIONS AND EQUATIONS}

In our studies of X-ray precursors, we approximate the inhomogeneities by shells which propagate ballistically, within boundaries of a cone. In order to illustrate in a simple analytical manner the basic relations between the properties of precursors and non-thermal flares, we assume that the shells have equal masses and proper widths, denoted as $\lambda_{0}$. We also assume that the conical angle, $\theta_{j}$, is sufficiently small that the kinematics and dynamics of ejecta can be described using a one-dimensional model. With the former assumption, the efficiency of the energy dissipation in the colliding shells is maximized, and, therefore, the magnitude of the bulk-Compton effects will be somewhat underestimated. Regarding the second assumption: the one-dimensional approach can directly be applied for models with very narrow jets and, in numerical models, it is generalized for a wider jets by summing radiative contributions from the $\delta \theta \ll \theta_{j}$ shell segments.

\subsection{Bulk-Compton luminosity}

Cold electrons, carried by two neighboring relativistic shells through the external diffuse radiation field with their respective bulk Lorentz factors $\Gamma_{2}>\Gamma_{1} \gg 1$, upscatter the ambient photons with energy $h \nu_{\text {diff }}$ up to energies

$$
h \nu_{B C} \simeq \Gamma_{i} \mathcal{D}_{i} h \nu_{\text {diff }} \quad i=1,2
$$


where

$$
\mathcal{D}_{i} \equiv \frac{1}{\Gamma_{i}\left(1-\beta_{i} \cos \theta_{\text {obs }}\right)},
$$

and $\theta_{o b s}$ is the angle between the line of sight and the jet axis. The scattered photons form the so-called bulk Compton radiation. That radiation is beamed into the jet direction giving the isotropic luminosity

$$
L_{B C, i} \equiv 4 \pi \frac{\partial L_{B C, i}}{\partial \Omega_{\vec{n}_{o b s}}} \simeq \int_{r_{0}}^{r_{s h}}\left(\frac{\partial N_{e, i}}{\partial r}\right)_{o b s} \frac{d E_{s c, i}^{\prime}}{d t^{\prime}} \mathcal{D}_{i}^{4} d r
$$

where $r_{0}$ is the distance at which the jet is fully formed and accelerated; $r_{s h}$ is the distance of the discontinuity surface which separates fluids of the two colliding shells;

$$
\frac{d E_{s c, i}^{\prime}}{d t^{\prime}}=\frac{d E_{s c, i}}{d t}=\frac{4}{3} \Gamma_{i}^{2} c \sigma_{T} u_{d i f f}
$$

is the amount of the scattered radiation energy per unit time, where $u_{\text {diff }}$ is the energy density of the external radiation field; and $\left(\partial N_{e, i} / \partial r\right)_{o b s} d r$ is the number of electrons contributing to the observed radiation at a given instant from a distance range $d r$. For a uniform radial distribution of electrons within the range $r_{r e a r}^{(o b s)}<r<r_{\text {front }}^{(o b s)}$

$$
\left(\frac{\partial N_{e, i}}{\partial r}\right)_{o b s}=\frac{N_{e}}{\lambda_{0} \mathcal{D}_{i}}
$$

and otherwise is zero. Note that the above is due to the fact that the source is observed as being stretched by a Doppler factor, i.e. that $r_{\text {front }}^{(\text {obs })}-r_{\text {rear }}^{(\text {obs }}=\lambda_{0} \mathcal{D}_{i}$.

Using Eqs. (3)-(5), one can find that for $u_{d i f f}=$ const up to a given distance $r_{m}$ and then falling very rapidly beyond $r_{m}$, the maximum bulk Compton luminosity, $L_{B C}$, is

$$
L_{B C, i} \simeq \frac{4}{3} c \sigma_{T} u_{d i f f} \Gamma_{i}^{2} \mathcal{D}_{i}^{4} N_{e} \epsilon_{i}
$$

where

$$
\epsilon_{i}=\operatorname{Min}\left[1 ; \frac{r_{m}}{\lambda_{0} \mathcal{D}_{i}}\right]
$$

\subsection{Number of electrons}

The number of cold electrons carried by a pair of shells is equal to the number of relativistic electrons involved in the production of non-thermal flares. Assuming that relativistic electrons are injected with a single power law distribution, $Q=K \gamma^{-p}$, the number of relativistic electrons injected during the collision time scale, $t_{\text {coll }}^{\prime}=\mathcal{D} t_{f l}$, is

$$
N_{e, i n j}=t_{f l} \mathcal{D} \int_{\gamma_{\min }}^{\gamma_{\max }} Q d \gamma=\frac{t_{f l} \mathcal{D} K}{(p-1) \gamma_{\min }^{p-1}}=2 N_{e}
$$


where $t_{f l}$ is the time scale of the non-thermal flare and $p>1$.

The normalization factor $K$ of the injection function $Q$ can be found from the formula for emissivity which in the $\gamma$-ray band is very likely dominated by Comptonization of external diffuse radiation fields by ultra-relativistic electrons (see, e.g., review by Sikora \& Madejski 2001). At a given frequency we have

$$
\nu L_{\nu} \simeq \frac{3}{8} \frac{\mathcal{D}^{6}}{\Gamma^{2}}\left[N_{\gamma} \gamma\right]|\dot{\gamma}|_{E C} m_{e} c^{2}
$$

where $N_{\gamma} \equiv \partial N_{e} / \partial \gamma$ is the differential energy distribution of electrons and $|\dot{\gamma}|_{E C}$ is the rate of the radiative losses of electrons due to the external Compton process.

Radiation observed in the $\mathrm{MeV}-\mathrm{GeV}$ band is produced by electrons which cool on time scales much shorter than the dynamical time scale (i.e. in the so-called fast cooling regime). For them, $N_{\gamma} \simeq \int_{\gamma} Q d \gamma /|\dot{\gamma}|_{E C}$, and inserting this into the Eq. (9) gives

$$
K=\frac{8(p-1)}{3 m_{e} c^{2} \gamma_{E}^{-p+2}}\left(\nu_{E} L_{\nu_{E}}\right) \frac{\Gamma^{2}}{\mathcal{D}^{6}},
$$

where $\gamma_{E} \simeq \mathcal{D} \sqrt{\nu_{E} / \nu_{\text {diff }}}$ and $\nu_{E}$ is the frequency at which the $\gamma$-ray luminosity is measured.

For $p \simeq 2$, which corresponds with the typical value of the energy flux spectral index in the EGRET band $\alpha \sim 1$ (Pohl et al. 1997),

$$
K \simeq \frac{8\left(\nu_{E} L_{\nu_{E}}\right)}{3 m_{e} c^{2}} \frac{\Gamma^{2}}{\mathcal{D}^{6}}
$$

and using Eq. (8) we obtain

$$
N_{e} \simeq \frac{\mathcal{D} t_{f l} K}{2 \gamma_{\min }} \simeq \frac{4 t_{f l}\left(\nu_{E} L_{\nu_{E}}\right)}{3 m_{e} c^{2}} \frac{\Gamma^{2}}{\mathcal{D}^{5}} \frac{1}{\gamma_{\min }}
$$

where $\gamma_{\min } \leq \mathrm{a}$ few, as indicated by power-law X-ray spectra extending down to energies $\leq 1 \mathrm{keV}$ (Tavecchio et al. 2000) and by circular polarization measurements (Wardle et al. 1998).

It is worthwhile to note that the number of electrons in cold shells could, in general, be much lower than that implied by the non-thermal flares, since a pair production via absorption of highest energy $\gamma$-rays in the $\gamma \gamma \rightarrow e^{+} e^{-}$process is always possible in principle. However, the external radiation Compton models predict the $\gamma$-ray spectra of quasars having the intrinsic cutoff at energies not much larger than $10 \mathrm{GeV}$ (see, e.g. Sikora et al. 1994). This together with characteristic $\gamma$-ray spectral slopes $\alpha \sim 1$ indicates that the number of pairs produced in the shock cannot significantly exceed the number of the primary electrons and 
positrons in the ejecta. It also should be mentioned here that in general population of relativistic particles in the shocked plasma can be accompanied by population of (quasi-)thermal particles. In such a case, the total number of electrons/positrons would be larger and the precursors would be more luminous than presented in $\S 3$, leading to stronger constraints on the internal shock models discussed in $\S 4$.

\subsection{The shell width}

In the internal shock model, the time scale of the non-thermal flares is related to the time scale of the shock operation. The latter is equal to the time scale of collision which depends on the velocities of the shells and on their widths. Since internal shocks are only marginally relativistic, the shell width can be derived by using the formula which ignores the width of the shocked plasma layer. In the shell rest frame this width is

$$
\lambda_{0}=\lambda_{0}^{\prime} \Gamma_{1,2}^{\prime}=\operatorname{ct}_{f l} \mathcal{D}\left|\beta_{1,2}^{\prime}\right| \Gamma_{1,2}^{\prime} \simeq c t_{f l} \mathcal{D} \frac{\alpha_{\Gamma}-1}{2 \sqrt{\alpha_{\Gamma}}}
$$

where $\left|\beta_{1,2}^{\prime}\right|=\beta_{2}^{\prime}=-\beta_{1}^{\prime}=\left(\alpha_{\Gamma}-1\right) /\left(\alpha_{\Gamma}+1\right), \Gamma_{1,2}^{\prime}=\Gamma_{2}^{\prime}=\Gamma_{1}^{\prime}=\left(\alpha_{\Gamma}+1\right) /\left(2 \sqrt{\alpha_{\Gamma}}\right)$, and $\alpha_{\Gamma} \equiv \Gamma_{2} / \Gamma_{1}$ (see Appendix A.2). Comparison of the approximate value of $\lambda_{0}$ given by Eq. (13) with its exact value is provided in Appendix A.3. It can be verified using formula (A20) that our approximation leads to an overestimate of $\lambda_{0}$ by no more than a factor 1.4. We use the approximate formula in order to calculate self-consistently the light curves, which, in addition to the precursors, include the non-thermal flares. The latter are computed by the BLAZAR code (Moderski, Sikora, \& Błażejowski 2003) which uses thin shell approximation.

\section{RESULTS}

The EGRET instrument detected many prominent flares in blazars (von Montigny et al. 1995). In the $30 \mathrm{MeV}-10 \mathrm{GeV}$ band, their apparent luminosities reach values $\nu L_{\nu} \sim 10^{48}$ $\mathrm{erg} \mathrm{s}^{-1}$, and their time scales, $t_{f l}$, are on the order of a few days. Relating $t_{f l}$ to the shock life-time, one can find that the distance range of the shock operation is

$$
\Delta r_{s h}=\frac{\beta c t_{f l}}{1-\beta \cos \theta_{o b s}} \equiv \beta c t_{f l} \mathcal{D} \Gamma \simeq 7.8 \times 10^{17}\left(\frac{t}{3 \text { day }}\right)\left(\frac{\Gamma}{10}\right)^{2} \mathrm{~cm} .
$$

Roughly symmetrical profiles of the observed non-thermal flares suggest that they occur at distances $r_{s h} \sim \Delta r_{s h}$ (see Sikora et al. 2001). At such distances, the energy density of the external diffuse radiation field is dominated by broad emission lines (BEL). From 
reverberation mapping of the BEL regions in AGNs and quasars we know that these lines are produced at

$$
r_{B E L} \sim 10^{18}\left(\frac{L_{U V}}{10^{46} \operatorname{erg~s}^{-1}}\right)^{0.7} \mathrm{~cm}
$$

(Kaspi et al. 2000).

Energy density of BEL radiation at $r<r_{B E L}$ is

$$
u_{\mathrm{BEL}} \simeq \frac{L_{\mathrm{BEL}}}{4 \pi c r_{\mathrm{BEL}}^{2}} \simeq 2.7 \times 10^{-3}\left(\frac{\xi_{\mathrm{BEL}}}{0.1}\right)\left(\frac{L_{U V}}{10^{46} \mathrm{erg} \mathrm{s}^{-1}}\right)^{-0.4} \mathrm{erg} \mathrm{cm}^{-3}
$$

where $\xi_{\mathrm{BEL}}=L_{\mathrm{BEL}} / L_{\mathrm{UV}}$. At $r>r_{\mathrm{BEL}}$ the energy density of BEL radiation drops faster than $r^{-2}$ and, therefore, in the first approximation, the production of the bulk Compton radiation at such distances can be ignored. Another effect which in the first approximation can be neglected arises due to the decreasing number of cold electrons due to the collision and shock formation. With the above approximations, one can estimate the maximum bulk Compton luminosities using Eq. (6) for $r_{m}=r_{B E L}$. The results are presented in Fig. 1, which shows the dependence of the precursor luminosities on the bulk Lorentz factor of the shocked plasma, and in Fig. 2, which illustrates the dependence of the luminosities on the angle of the jet with respect to our line of sight. In both Figures, we plot separately the value of $L_{B C}$ for each of the two shells for various values of $\alpha_{\Gamma}$ (the ratio of Lorentz factors of the two shells); both shells will subsequently undergo a collision, which will result in the non-thermal flare. The values of $L_{B C}$ plotted there are calculated by combining Eqs. (6), (7), (12), and (13), which give $L_{B C, i} \propto \Gamma_{i}^{2} \Gamma^{2} \mathcal{D}_{i}^{4} / \mathcal{D}^{5}$ for $\epsilon_{i}=1$ (entire source is contributing to the observed radiation) and $L_{B C, i} \propto \Gamma_{i}^{2} \Gamma^{2} \mathcal{D}_{i}^{3} / \mathcal{D}^{6}$ for $\epsilon_{i}<1$.

It is apparent from Fig. 1 that the luminosity of the precursors produced by the faster of the two shells becomes much larger than that due to the slower shell when the bulk Lorentz factor decreases. The large difference at small $\Gamma$ factors reflects the stronger Doppler boosting of radiation from the faster moving sources than from the slower ones. The decrease of this difference with increasing $\Gamma$ is caused mainly by a large reduction of the beaming factor of the faster shell for the observer located at fixed angle outside the Doppler cone.

Fig. 2 shows that bulk luminosities from faster moving shells have lowest values at $\theta_{o b s} \sim 1 / \Gamma$. For smaller $\theta_{o b s}$, the shape of $L_{B C, 2}\left(\theta_{o b s}\right)$ scales roughly with $\mathcal{D}_{2}^{3}$; for larger $\theta_{o b s}$, the increase of bulk Compton luminosity is mainly due to increase of a number of electrons required to produced a given luminosity of non-thermal flares. Since for $\theta_{o b s}<1 / \Gamma$ the dependence of $\mathcal{D}_{1}$ on $\theta_{o b s}$ is very flat, the shape of $L_{B C, 1}\left(\theta_{o b s}\right)$ is determined by a factor $\mathcal{D}^{-5}$ and, therefore, $L_{B C, 1}$ increases with the viewing angle. This increase continues at larger angles, but is diminished due to the term $\mathcal{D}_{1}^{4}$, which starts to contribute more strongly to 
the dependence of the $L_{B C, 1}$ on the angle of view for $\theta_{o b s}>1 / \Gamma_{1}$. Since the bulk Compton luminosities presented on Figs. 1 and 2 are calculated for $\theta_{j}=0$, while real sub-parsec jets are expected to have $\theta_{j} \sim 1 / \Gamma$, the very high values of $L_{B C, 2}$ at $\theta_{\text {obs }}<1 / \Gamma$ are probably rather unrealistic.

The light-curves of soft X-ray precursors and of non-thermal flares are presented in Fig. 3 - for jets with $\theta_{j} \ll \Gamma_{2}$, and in Fig. 4 and 5 - for jets with $\theta_{j}=\theta_{o b s}=1 / \Gamma$. These light curves have been calculated for: $u_{\text {diff }} \propto r^{-3}$ for $r<r_{d}=3 \times 10^{16} \mathrm{~cm} ; u_{\text {diff }}=u_{B E L}$ for $r_{d}<r<r_{B E L}$; and $u_{d i f f} \sim r^{-2.5}$ for $r>r_{B E L}$. The first branch mimics the contribution of the seed photons for the EC process from the accretion disk (Dermer \& Schlickeiser 2002). This dominates radiative energy losses of relativistic electrons at $r<r_{d}$. The latter branch represents the extension of BEL emissivities beyond the distance $r_{B E L}$. The time profiles of the non-thermal flares for $\theta_{j}=1 / \Gamma$ are computed using the code BLAZAR described in Moderski, Sikora, \& Błażejowski (2003).

It is apparent from Fig. 3 and from the left panels of Figs. 4 and 5 that precursors produced by slower shells are very extended in time and reach their maximum luminosities significantly before the emergence of non-thermal flares. In contrast, the brighter precursors, presumably produced by the faster shells, overlap significantly with the non-thermal flares, and, for $\theta_{o b s} \leq 1 / \Gamma$, their peaks only marginally precede the peaks of non-thermal flares. (see right panels of Figs. 4 and 5). More significant feature of the brighter precursors is much faster decay of their light curves than of non-thermal flares.

In all Figures except for Fig. 3, the cold shells are assumed to be formed at a distance $r_{d}$, above which energy density of external radiation field as measured in a jet comoving frame is dominated by the broad emission line radiation. In Fig. 3, the shells are assumed to be formed in the close proximity to the central engine and the light-curves plotted in those Figures include both Comptonization of broad emission lines and Comptonization of the accretion disk radiation. Comptonization of the disk radiation produces additional "bumps" in the light curves of the precursors, with a sharp drop at the time corresponding to $r_{d}$. This sudden drop is a result of the apparent lengths of the shells being stretched by relativistic effects. For the parameters from Figure 3 the apparent length of the shell is much larger than the size of the region in which radiation from the disk dominates, while the observed travel time through the region is very short, since it is relativistically reduced by a factor $\left(1-\beta_{i} \cos \theta_{\text {obs }}\right)$. This results in a nearly rectangular shape of the part of the precursor lightcurve. Figure 3 clearly shows that the Comptonization of the disk radiation provides about three times larger luminosity than Comptonization of broad emission lines, as indicated by maximum amplitude of the precursors in both cases. 


\section{DISCUSSION AND CONCLUSIONS}

If the $\gamma$-ray flares observed in many blazars are produced via internal shocks, they should be accompanied by soft X-ray precursors. The precursors are predicted to result from Comptonization of external UV continuum and broad emission line photons by cold electrons carried by the relativistic ejecta. The ejecta, moving with different velocities, collide with each other and form shocks where relativistic electrons are accelerated. The dynamics of such events is very complex, and depends on the unknown structure and kinematics of the ejecta. However, some basic properties, such as minimal luminosities and profiles of the light curves of precursors vs. those of non-thermal flares should not depend strongly on the details of the model. We study them by approximating the ejecta by discrete, intrinsically identical shells. We analyze the dependence of precursors on various parameters such as the bulk Lorentz factor of the shocked plasma, $\Gamma$, the ratio of the bulk Lorentz factors of shells, $\alpha_{\Gamma}=\Gamma_{2} / \Gamma_{1}$, and the viewing angle, $\theta_{o b s}$.

Our results show that precursors produced by faster shells are typically 10 - 30 times less luminous than $\gamma$-ray flares if the shells are formed at $r_{0}=r_{d}$, and about 10 times less luminous if the shells are formed much closer to the central engine and contribution from the Comptonization of the disk radiation is included. We note here that our model predicts that the low energy tail of the EC component of the non-thermal flare contributes in the soft X-ray band about 30 - 100 less than at the peak (see, e.g., Moderski, Sikora, \& Błażejowski 2003). The Synchrotron Self-Compton (SSC) contribution is also predicted to be of the same order as EC component. With this, the precursors from the faster shells are predicted to dominate in the soft X-ray band over the non-thermal flares, even if the contribution from the accretion disk is not included.

As shown in Figures 4 and 5, the brighter precursors overlap significantly in time with non-thermal flares, but because their flux should decay much faster than for the non-thermal flares, they should be easily distinguished from the synchrotron and SSC contributions, provided they are monitored with the intra-day time resolution. Furthermore, presence of precursors can be verified by future polarimetric measurements. As predicted by Begelman \& Sikora (1987), the bulk Compton radiation should be highly polarized, with the electric vector perpendicular to the jet axis. The polarized soft X-ray radiation is expected to be produced also by synchrotron and SSC process. However, for magnetic fields dominated by the transverse component (due to compression of chaotic magnetic fields in the transverse shocks), that radiation should be polarized with the electric vector parallel to the jet axis. Hence, the polarization position angle measurements and the variability analysis can provide two independent diagnosis of the bulk-Compton radiation.

Clearly, search for the soft X-ray precursors is a viable avenue to study the nature 
of blazar jets, and in particular, to determine if their kinetic luminosities are dominated by energy flux of particles or by Poynting flux. The lack of observational evidence for precursors by future observations will indicate that cold inhomogeneities originate in situ, at distances just prior to the formation of shocks, rather then resulting from modulation of the outflow by a central engine. Their formation may be related to the transition from the Poynting flux to matter dominated flow; then, the locally fast moving inhomogeneities can be a product of intensive magnetic field reconnection events. Another possibility is that the nonthermal flares are produced by relativistic electrons directly accelerated in the reconnection sites. However, this scenario can be questioned by the fact that the spectra of injected electrons predicted theoretically and numerically are much narrower than deduced from spectral analysis of the high energy non-thermal flares (Zenitani \& Hoshino 2001; Larrabee, Lovelace, \& Romanowa 2003). In any case, given the promising future of the $\mathrm{MeV} / \mathrm{GeV}$ $\gamma$-ray astronomy with the upcoming launch of the GLAST mission, this question will be addressed directly by observations. However, it is essential that simultaneous observations in the soft X-ray band are conducted as well, although the lack of a sensitive all-sky X-ray monitor will limit studies to probably a limited number of pointed observations in the X-ray band by satellites such as Chandra, XMM-Newton, and Astro-E2.

This project was partially supported by Polish KBN grants 5 P03D 00221 and PBZKBN-054/P03/2001, by Chandra grants no. GO1-2113X and GO4-5125X from NASA via Smithsonian Astrophysical Observatory, and by the Department of Energy contract to SLAC no. DE-AC3-76SF00515. R.M. and M.S. thank SLAC for its hospitality during the completion of part of this work. We thank the anonymous referee for her/his valuable comments that helped to improve this paper.

\section{A. APPENDIX}

\section{A.1. Velocity of the shocked plasma and efficiency of the energy dissipation}

Momentum and energy conservation for collision of two intrinsically identical shells gives the velocity of the shocked plasma

$$
\beta=\frac{\beta_{1} \Gamma_{1}+\beta_{2} \Gamma_{2}}{\beta_{1}+\beta_{2}},
$$

and the efficiency of energy dissipation

$$
\eta_{d i s s}=1-\frac{2 \Gamma}{\Gamma_{1}+\Gamma_{2}},
$$


where $\Gamma=1 / \sqrt{1-\beta^{2}}$.

For $\Gamma_{2}$ and $\Gamma_{1} \gg 1$

$$
\begin{gathered}
\beta \simeq 1-\frac{1}{2 \Gamma_{1} \Gamma_{2}}, \\
\Gamma \simeq \frac{1}{\sqrt{1-\beta^{2}}} \simeq \sqrt{\Gamma_{1} \Gamma_{2}}
\end{gathered}
$$

and

$$
\eta_{\text {diss }} \simeq \frac{\left[\left(\Gamma_{2} / \Gamma_{1}\right)^{1 / 2}-1\right]^{2}}{\left(\Gamma_{2} / \Gamma_{1}\right)+1}
$$

For a given $\Gamma$ and $\alpha_{\Gamma} \equiv \Gamma_{2} / \Gamma_{1}$, we have $\Gamma_{1} \simeq \Gamma / \sqrt{\alpha_{\Gamma}}$ and $\Gamma_{2} \simeq \Gamma \sqrt{\alpha_{\Gamma}}$.

\section{A.2. Shell velocities in the frame of the shocked plasma}

From Lorentz transformation of shell velocity from the central engine frame to the shocked plasma frame

$$
\beta_{i}^{\prime}=\frac{\beta_{i}-\beta}{1-\beta_{i} \beta}
$$

For $\Gamma_{i}$ and $\Gamma \gg 1$ it gives

$$
\begin{aligned}
& \beta_{i}^{\prime} \simeq \frac{\Gamma_{i}^{2}-\Gamma^{2}}{\Gamma_{i}^{2}+\Gamma^{2}}, \\
& \Gamma_{i}^{\prime} \simeq \frac{\Gamma_{i}^{2}+\Gamma^{2}}{2 \Gamma_{i} \Gamma} .
\end{aligned}
$$

For $\Gamma \simeq \sqrt{\Gamma_{1} \Gamma_{2}}$ the above formulae give

$$
\Gamma_{1}^{\prime}=\Gamma_{2}^{\prime}=\frac{1}{2} \frac{\alpha_{\Gamma}+1}{\sqrt{\alpha_{\Gamma}}},
$$

and

$$
\beta_{2}^{\prime}=-\beta_{1}^{\prime}=\frac{\alpha_{\Gamma}-1}{\alpha_{\Gamma}+1}
$$

\section{A.3. The shock width}

From the shock theory, the ratio of the downstream density $n_{d s}$ to the upstream density $n_{u s}$ is

$$
\frac{n_{d s}}{n_{u s}}=\frac{\hat{\gamma} \Gamma_{u s}^{\prime}+1}{\hat{\gamma}-1},
$$


where $\Gamma_{u s}^{\prime}=\Gamma_{1,2}^{\prime}$ is the bulk Lorentz factor of the upstream flow as measured in the downstream (shocked plasma) frame, and $5 / 3<\hat{\gamma}<4 / 3$ (see Blandford \& McKee 1976). From particle number continuity

$$
\frac{\lambda_{0}}{\lambda} \simeq \frac{n_{d s}}{n_{u s}},
$$

where $\lambda_{0}$ is the proper width of the unshocked shell, and $\lambda$ is the proper width of the shocked plasma layer. In the downstream frame (corresponding to that of the shocked plasma/discontinuity surface), time of the collision $t_{\text {coll }}^{\prime}$ equals the time necessary for the shock front to travel the distance $\lambda$

$$
t_{\text {coll }}^{\prime}=\frac{\lambda}{c\left|\beta_{s}^{\prime}\right|},
$$

where $\beta_{s}^{\prime}$ is the velocity of the shock front. In the shocked plasma frame, the width of the unshocked plasma shell is $\lambda_{0} / \Gamma_{u s}^{\prime}$ and thus during the collision, the outer boundary of the unshocked plasma travels the distance

$$
\lambda_{0} / \Gamma_{u s}^{\prime}-\lambda=t_{c o l l}^{\prime} c\left|\beta_{u s}^{\prime}\right|,
$$

where $\left|\beta_{u s}^{\prime}\right|=\left|\beta_{1,2}^{\prime}\right|$ is the velocity of the upstream flow in the reference frame of the shocked plasma.

From above equations

$$
\begin{aligned}
& \left|\beta_{s}^{\prime}\right|=\left|\beta_{u s}^{\prime}\right| \frac{(\hat{\gamma}-1) \Gamma_{u s}^{\prime}}{\Gamma_{u s}^{\prime}+1}, \\
& \lambda=c t_{c o l l}^{\prime}\left|\beta_{s}^{\prime}\right|=c t_{f l} \mathcal{D} g,
\end{aligned}
$$

and

$$
\lambda_{0}=\frac{\hat{\gamma} \Gamma_{u s}^{\prime}+1}{\hat{\gamma}-1} \lambda=c t_{f l} \mathcal{D} g_{0},
$$

where

$$
g=\frac{(\hat{\gamma}-1)\left|\beta_{u s}^{\prime}\right| \Gamma_{u s}^{\prime}}{\Gamma_{u s}^{\prime}+1},
$$

and

$$
g_{0}=\frac{\left(\hat{\gamma} \Gamma_{u s}^{\prime}+1\right)\left|\beta_{u s}^{\prime}\right| \Gamma_{u s}^{\prime}}{\Gamma_{u s}^{\prime}+1} .
$$

Comparison of the exact value of the shell width given by Eq. (A17) with its approximate value given by Eq. (13) gives

$$
\frac{\lambda_{0}^{(\text {approx })}}{\lambda_{0}^{(\text {exact })}}=\frac{\hat{\gamma} \Gamma_{u s}^{\prime}+1}{\Gamma_{u s}^{\prime}+1} .
$$




\section{A.4. The pair content}

Amount of energy needed to be injected into relativistic electrons to produce the observed non-thermal flares is

$$
E_{e, i n j}^{\prime}=t_{c o l l}^{\prime} L_{e, i n j}^{\prime}=t_{f l} \mathcal{D} \int_{\gamma_{\min }}^{\gamma_{\max }} Q m_{e} c^{2} \gamma d \gamma
$$

where $Q$ is determined from the observed spectra (see $\S 2.2$ ). This energy cannot exceed the amount of energy dissipated during the collision of two shells, which is

$$
E_{\text {diss }}^{\prime}=\frac{\left(E_{1}+E_{2}\right) \eta_{\text {diss }}}{\Gamma}
$$

where for $n_{p} m_{p} \gg n_{e} m_{e}$

$$
E_{1}+E_{2} \simeq N_{p} m_{p} c^{2}\left(\Gamma_{1}+\Gamma_{2}\right)
$$

Hence, for a given amount of electrons determined from observations of non-thermal flares, the condition $E_{d i s s}^{\prime}>E_{e, i n j}^{\prime}$ gives the minimum number of protons in shells, and, respectively, the maximum pair content. Parameterizing the fraction of dissipated energy tapped by relativistic electrons by $\eta_{e}$, and noting that the number of electrons per shell is $N_{e}=0.5 N_{e, i n j}=0.5 t_{f l} \mathcal{D} \int Q d \gamma$, we obtain

$$
\frac{N_{e}}{N_{p}}=\eta_{e} \frac{m_{p}}{m_{e}} \frac{\left(\sqrt{\Gamma_{2} / \Gamma_{1}}-1\right)^{2}}{2 \sqrt{\Gamma_{2} / \Gamma_{1}}} \frac{1}{\bar{\gamma}_{i n j}},
$$

where $\bar{\gamma}_{i n j} \equiv \int Q \gamma d \gamma / \int Q d \gamma$ is the averaged energy of injected electrons. Since $n_{e} \equiv n_{+}+n_{-}$ and $n_{p}+n_{+}=n_{-}$(charge neutrality), the pair content (number of pairs per proton) is $n_{+} / n_{p}=\left(\left(N_{e} / N_{p}\right)-1\right) / 2$.

\section{REFERENCES}

Asada, K., Inoue, M., Yutaka, U. et al. 2002, PASJ, 54, L39

Begelman, M.C., \& Sikora, M. 1987, ApJ, 322, 650

Blandford, R.D., \& McKee, C.F. 1976, Phys. Fluids 19, 1130

Contopoulos, J. 1995, ApJ, 450, 616

Dermer, C.D., \& Schlickeiser, R. 2002, ApJ, 575, 667

Enßlin, T.A. 2003, A\&A, 401, 499 
Gabuzda, D.C., \& Murray, É. 2003, astro-ph/0309668

Homan, D.C. 2004, astro-ph/0401320

Homan, D.C., Attridge, J.M., \& Wardle, J.F.C. 2001, ApJ, 556, 113

Kaspi, S., Smith, P.S., Netzer, H., et al. 2000, ApJ, 533, 631

Larrabee, D.A., Lovelace, R.V.E., \& Romanova, M.M. 2003, ApJ, 586, 72

Lovelace, R.V.E., Newman, W.I., \& Romanova, M.M. 1997, ApJ, 484, 628

Madejski, G.M., Sikora, M., \& Kamae, T. 2003, in "MAXI Workshop on AGN Variability" (Nikko, Japan, 10-11 March 2001), IPCR CR-124, ed. N. Kawai et al. (RIKEN: Saitama, Japan), 45 [astro-ph/0107393]

Moderski, R., Sikora, M., \& Błażejowski, M. 2003, A\&A, 406, 855

Pohl, M., Hartman, R., Jones, B.B., \& Srekumar, P. 1997, A\&A, 326, 51

Ruszkowski, M., \& Begelman, M.C. 2002, ApJ, 573, 485

Sikora, M., Begelman, M.C., \& Rees, M.J. 1994, ApJ, 421, 153

Sikora, M., Błażejowski, M., Begelman, M. C., \& Moderski, R. 2001, ApJ, 554, 1

Sikora, M., \& Madejski, G.M. 2000, ApJ, 534, 109

Sikora, M., \& Madejski, G.M. 2001, in High Energy Gamma-Ray Astronomy, ed. F.A. Aharonian \& H.J. Völk, AIP Conf. Proc., 558, 275 [astro-ph/0101382]

Sikora, M., \& Madejski, G.M. 2002, in Current High Energy Emission around Black Holes, ed. C.-H. Lee, \& H.-Y. Chang (New Jersey, London, Singapore, Hong Kong: World Scientific), 132 [astro-ph/0112231]

Spada, M., Ghisellini, G., Lazzati, D., \& Celotti, A. 2001, MNRAS, 325, 1559

Tavecchio, F., Maraschi, L., Ghisellini, G., et al. 2000, ApJ, 543, 535

Vlahakis, N., \& Königl, A. 2003, astro-ph/0310747

von Montigny, C., Bertsch, D.L., Chiang, J., et al. 1995, ApJ, 440, 525

Wardle, J.F.G., Homan, D.C., Ojha, R., \& Roberts, D.H. 1998, Nature, 395, 457

Zenitani, S., \& Hoshino, M. 2001, ApJ, 562, L63 
This page was intentionally left blank.

This preprint was prepared with the AAS LATEX macros v5.0. 


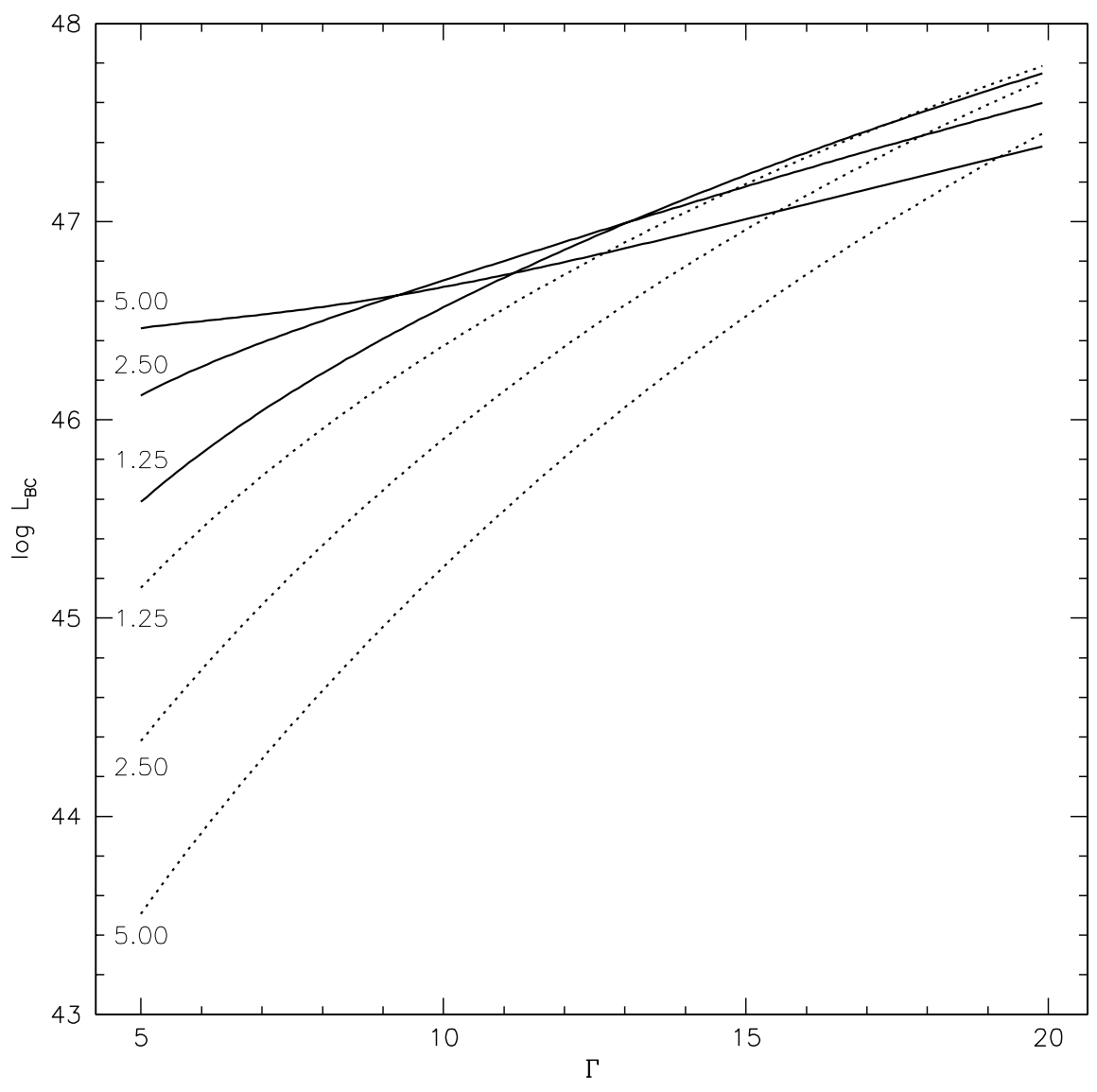

Fig. 1.- Precursor luminosity as a function of the bulk Lorentz factor $\Gamma$. The angle of view is $\theta_{\text {obs }}=1 / 10$, and the three pairs of curves are calculated for ratio of the Lorentz factors of the two shells $\alpha_{\Gamma}=1.25 ; 2.5$; and 5 (marked beside the curves on the left side of the plot). The solid lines are for faster of the two shells, the dotted lines are for the slower of the two shells. The models are calculated for: $u_{\text {diff }}=$ const $=2.7 \times 10^{-3} \mathrm{erg} \mathrm{cm}^{-3}$ for $r \leq r_{B E L}$ and $u_{\text {diff }}=0$ for $r>r_{B E L} ; \nu_{E} L_{\nu_{E}}=10^{48} \mathrm{erg} \mathrm{s}^{-1} ; t_{f l}=3 \mathrm{~d}$; and $\gamma_{\min }=1$. Jet is assumed to be very narrow $\left(\theta_{j} \ll 1 / \Gamma_{2}\right)$. 


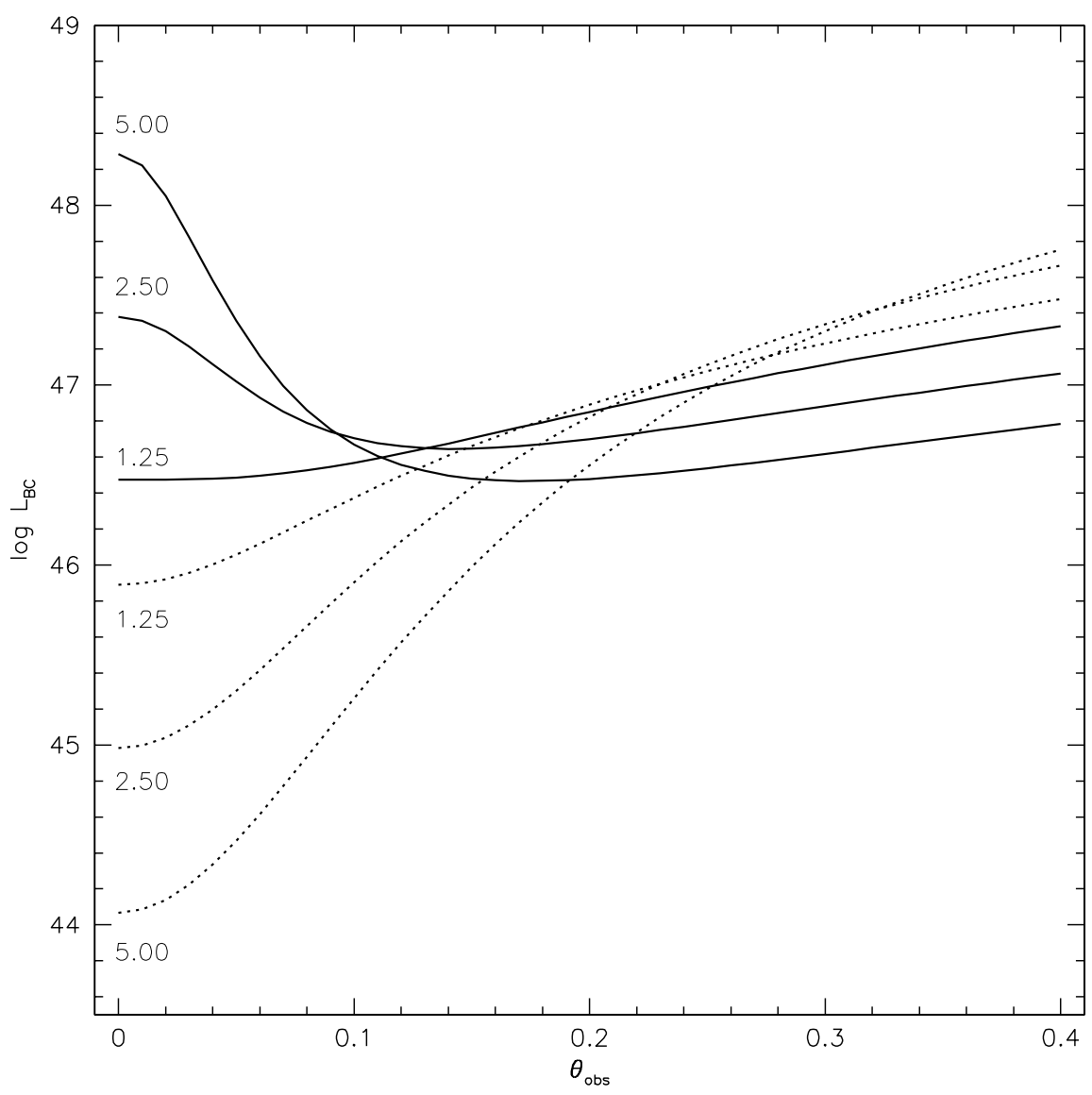

Fig. 2.- Precursor luminosity as a function of the angle of view $\theta_{o b s}$, for $\Gamma=10$. Other parameters are the same as in Figure 1. 

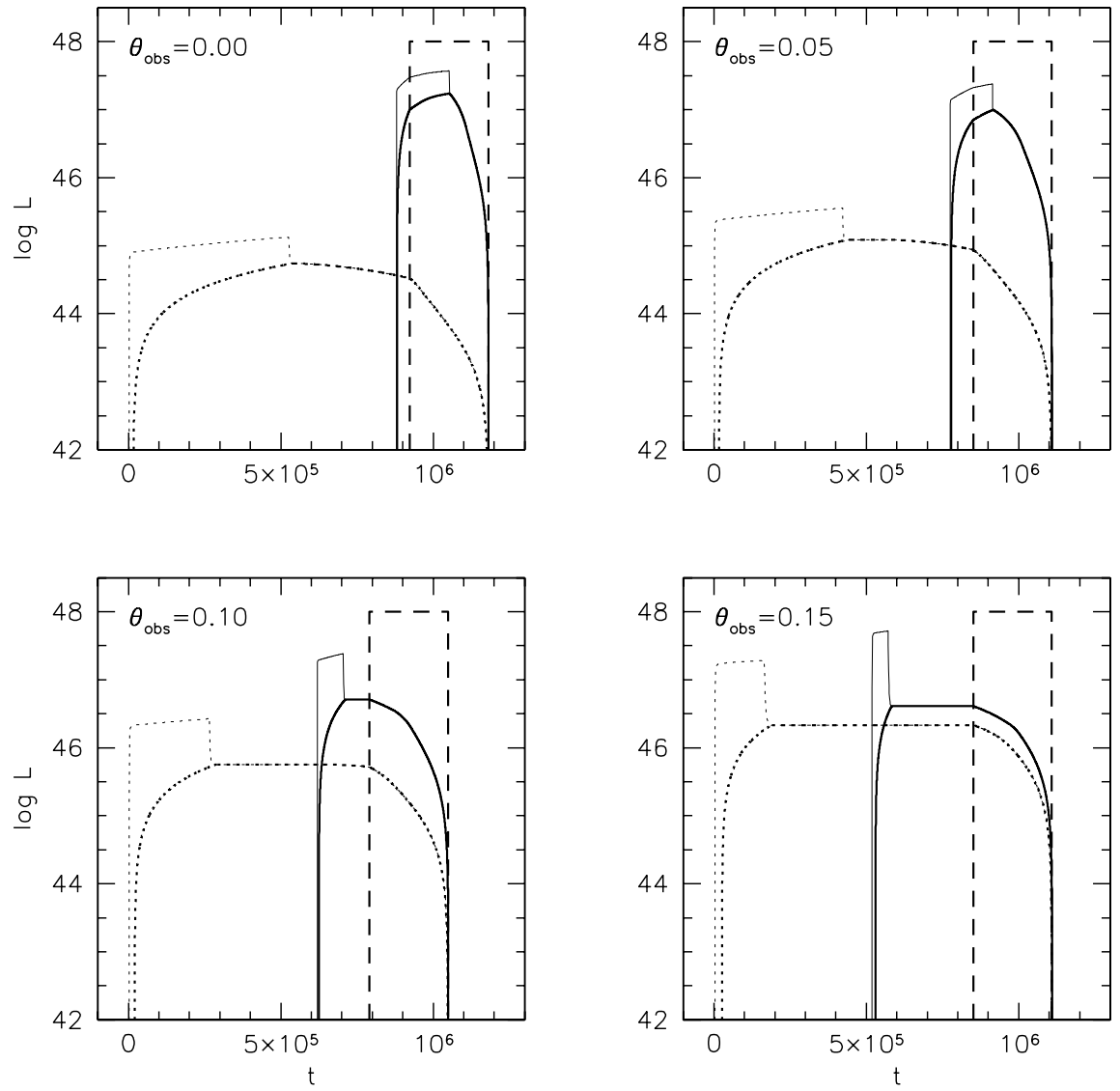

Fig. 3.- Light curves of precursors and flares for four different values of $\theta_{\text {obs }}$. The solid lines are the precursors produced by the faster shells; the dotted lines are the precursors produced by the slower shells; and the dashed lines are the non-thermal $\gamma$-ray flares as produced deeply in the fast cooling regime. The models are computed for $u_{\text {diff }}=$ const at $r_{d}<r<r_{B E L}$, and for the power-law distributions at $r<r_{d}$ and $r>r_{B E L}$ as described in the text. Precursors starting at $r_{0}=r_{d}=3 \times 10^{16} \mathrm{~cm}$ and $r_{0}=3 \times 10^{15} \mathrm{~cm}$ are shown by the thick and thin curves, respectively. Production of non-thermal flares by shocks starts at a distance $r_{0, s h}=7.8 \times 10^{17} \mathrm{~cm}$; the shocks terminate at a distance $r_{0, s h}+\Delta r_{s h}$ (see Eq. 14). The kinematical parameters are $\Gamma=10$ and $\alpha_{\Gamma}=3$. Other parameters are the same as in Figures 1 and 2 . 

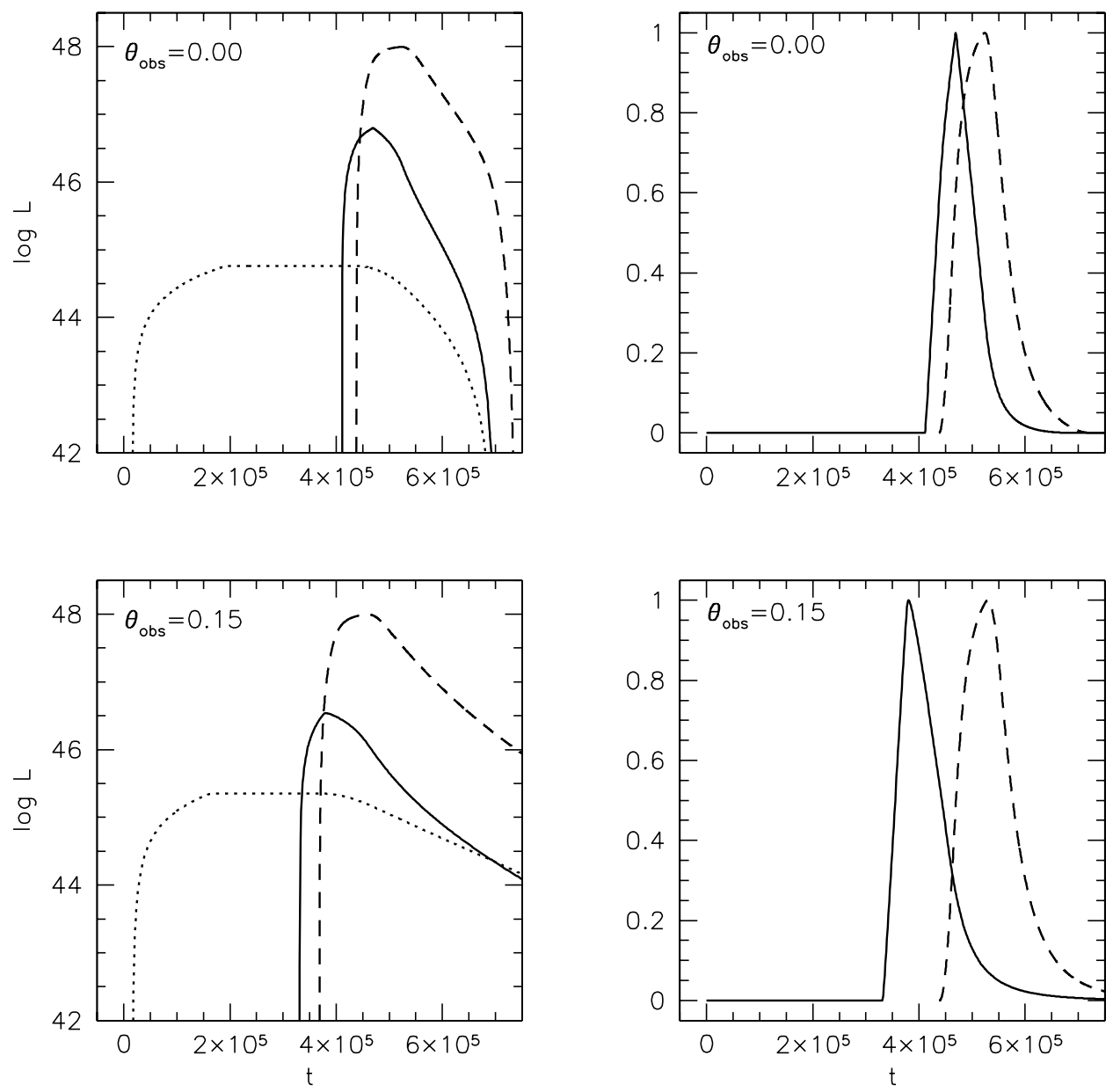

Fig. 4. - Light-curves of the two types of precursors and the non-thermal flares for $\theta_{\text {obs }}=0$ (the upper left panel) and $\theta_{o b s}=0.15$ (the lower left panel) for $\theta_{j}=1 / \Gamma=0.1$. Those due to the faster shells and the flares are re-drawn in linear scale on the right panels with their peaks normalized to one. The models are computed for: $t_{f l}=1 \mathrm{~d} ; r_{0, s h}=\Delta r_{s h}$; and $r_{0}=r_{d}$. Other parameters are the same as in Figure 3. 

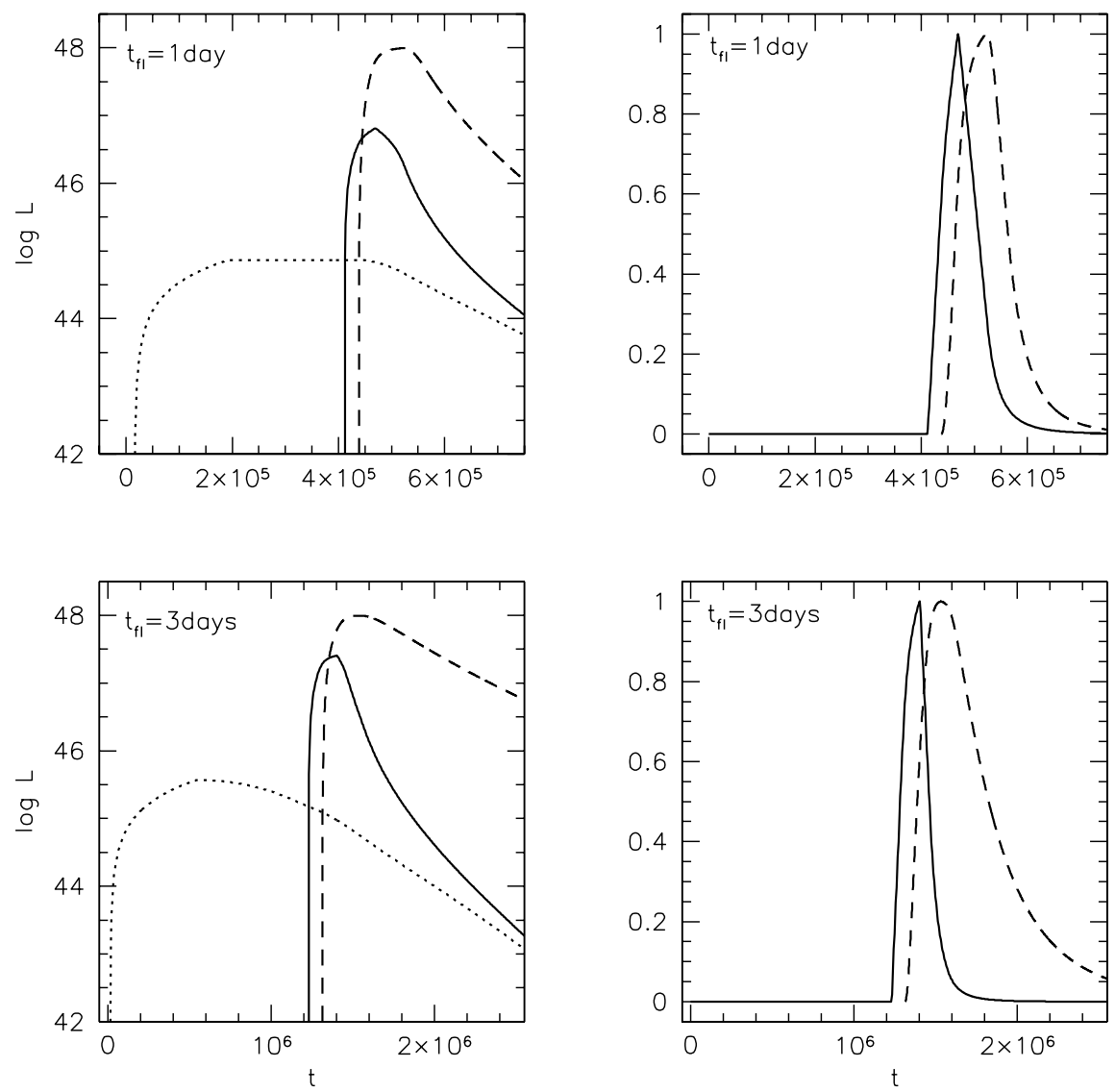

Fig. 5.- Comparison of light-curves computed for $t_{f l}=1$ day and 3 days. Models are computed for $\theta_{o b s}=1 / \Gamma=0.1$. All other parameters are the same as in Figure 4. 